\title{
WEIGHTS OF SEMI-INVARIANTS OF THE QUOTIENT DIVISION RING OF AN ENVELOPING ALGEBRA
}

\author{
E. NAUWELAERTS AND A. I. OOMS
}

(Communicated by Donald S. Passman)

\begin{abstract}
Let $L$ be a finite dimensional Lie algebra over a field $k$ of characteristic zero, $D(L)$ the quotient division ring of $U(L)$. It is shown that the weights of the semi-invariants of $D(L)$ form a finitely generated, free abelian group $\Lambda_{D}(L)$. It follows, among other things, that the semicenter of $D(L)$ is isomorphic to the group algebra of $\Lambda_{D}(L)$ over the center $Z(D(L))$ of $D(L)$.
\end{abstract}

Introduction. Throughout this paper $L$ will be a nonzero finite-dimensional Lie algebra over a field $k$ of characteristic zero. Let $U(L)$ be the universal enveloping algebra of $L$ with center $Z(U(L)), D(L)$ the division ring of quotients of $U(L)$ with center $Z(D(L))$. For each $\lambda \in L^{*}$ we denote by $D(L)_{\lambda}$ the set of those $u \in D(L)$ such that $\operatorname{ad} x(u)=\lambda(x) u$ for all $x \in L$. Its elements are called the semi-invariants of $D(L)$ relative to $\lambda$.

Clearly $D(L)_{\lambda} D(L)_{\mu} \subset D(L)_{\lambda+\mu}$ for all $\lambda, \mu \in L^{*}$. Put $U(L)_{\lambda}=U(L) \cap D(L)_{\lambda}$. The sum of the $D(L)_{\lambda}$ (resp. $\left.U(L)_{\lambda}\right)$ is direct and is a factorial subalgebra $S z(D(L)$ ) (resp. $S z(U(L))$ ) which is called the semicenter of $D(L)$ (resp. $U(L))[10,13]$. Let $\Lambda_{D}(L)$ (resp. $\Lambda(L)$ ) be the set of all weights $\lambda \in L^{*}$ such that $D(L)_{\lambda} \neq 0$ (resp. $\left.U(L)_{\lambda} \neq 0\right)$. Since any semi-invariant of $D(L)$ can be written as a quotient of two semi-invariants of $U(L), \Lambda_{D}(L)$ is the additive subgroup of $L^{*}$ generated by the semigroup $\Lambda(L)[18,6$, p. 329]. Using the fact that each subfield of $D(L)$ containing $k$ is finitely generated over $k$ we are able to prove that $\Lambda_{D}(L)$ is a finitely generated free abelian group. This result has some interesting consequences. For instance, the semicenter $S z(D(L))$ is isomorphic to the group algebra of $\Lambda_{D}(L)$ over $Z(D(L))$. In particular $S z(D(L))$ is Noetherian. The same need not be true however for $S z(U(L))$ (not even if $L$ is nilpotent). Also, it is shown that there exists a nonzero semi-invariant $e$ in $U(L)$ such that the localization $U(L)_{e}$ of $U(L)$ satisfies the Formanek condition, i.e. any nonzero ideal of $U(L)_{e}$ contains a nonzero central element of $U(L)_{e}$. Moreover, the center $Z\left(U(L)_{e}\right)$ is factorial and its quotient field coincides with $Z(D(L))$. Finally, let $\lambda_{1}, \ldots, \lambda_{r}$ be a Z-basis of $\Lambda_{D}(L)$. If $L$ is Frobenius then $\lambda_{1}, \ldots, \lambda_{r}$ are linearly independent over $k$ [6, Theorems 3.2 and 4.3]. The same result also holds if $L$ is an algebraic Lie algebra.

Part of this paper was reported at a meeting on enveloping algebras at Oberwolfach.

1. The main theorem and some consequences. We thank $R$. Rentschler for bringing the following result to our attention.

Received by the editors June 29, 1987 and, in revised form, September 21, 1987.

1980 Mathematics Subject Classification (1985 Revision). Primary 17B35; Secondary 16A08.

Key words and phrases. Finite-dimensional Lie algebra, universal enveloping algebra, semiinvariants. 
THEOREM 1.1 [19, THEOREM 3]. Let $D$ be a division algebra over a field $k$, $F$ a commutative subfield of $D$ containing $k$. Then $F$ is a finitely generated field extension of $k$ if and only if $D \otimes_{k} F$ is left and right Noetherian.

COROLLARY 1.2. (a) Let $F$ be a commutative subfield of $D(L)$ containing $k$. Then $F$ is a finitely generated field extension of $k$.

(b) $k$ is algebraically closed in $D(L)$; that is, if $a \in D(L)$ is algebraic over $k$ then $a \in k$.

PROOF. The proof is analogous with that of Theorem 4 of [19].

(a) $U(L) \otimes_{k} F$ is isomorphic to $U\left(L \otimes_{k} F\right)$ and hence is left and right Noetherian. The same holds for $D(L) \otimes_{k} F$ since it is the localization of $U(L) \otimes_{k} F$ at the Ore set $\{u \otimes 1 \mid u \in U(L), u \neq 0\}$. Finite generation of $F$ follows from Theorem 1.1.

(b) Let $a \in D(L)$ and put $F=k(a)$. As $D(L) \otimes_{k} F$ is a localization of $U\left(L \otimes_{k} F\right)$ (up to isomorphism), it is a domain. Since $F \otimes_{k} F$ embeds in $D(L) \otimes_{k} F$ it also is a domain. Now, if $a$ is algebraic over $k$, then $a \in k$ by [5, p. 248 ex. 5].

We can now prove the main result of this note.

THEOREM 1.3. $\Lambda_{D}(L)$ is a finitely generated free abelian group.

PROOF. Since $\Lambda_{D}(L)$ is a torsion free abelian group, it suffices to show that it is finitely generated. Let $F$ be the common quotient field of $S z(U(L))$ and $S z(D(L))$ [6, Corollary 1.11]. By (a) of the foregoing corollary, $F$ is a finite field extension of $k$. Hence there exist nonzero semi-invariants $u_{i} \in U(L)_{\mu_{i}}$ such that $F=k\left(u_{1}, \ldots, u_{s}\right)$ (we may even assume that the $u_{i}$ are irreducible). Let $G$ be the subgroup of $\Lambda_{D}(L)$ generated by $\mu_{1}, \ldots, \mu_{s}$. We claim that $G=\Lambda_{D}(L)$. Take any $\lambda \in \Lambda_{D}(L)$ and $w \in D(L)_{\lambda}$ such that $w \neq 0$.

We have to show that $\lambda \in G$. We can find nonzero elements $u, v \in k\left[u_{1}, \ldots, u_{s}\right]$ such that $w=u v^{-1}$. Hence $u=\sum_{m} a_{m} u_{1}^{m_{1}} \cdots u_{s}^{m_{s}}$ where $a_{m} \in k$ and $m=$ $\left(m_{1}, \ldots, m_{s}\right) \in \mathbf{N}^{s}$. Clearly $a_{m} u_{1}^{m_{1}} \cdots u_{s}^{m_{s}} \in U(L)_{\mu}$ where $\mu=m_{1} \mu_{1}+\cdots+$ $m_{s} \mu_{s} \in G$. After regrouping the terms according to their weights, we obtain $u=\sum x_{\alpha}, \alpha \in G$ and $x_{\alpha} \in U(L)_{\alpha}$. Similarly, we have $v=\sum y_{\beta}, \beta \in G$ and $y_{\beta} \in U(L)_{\beta}$, not all zero. Next, we observe that

$$
\sum w y_{\beta}=w v=u=\sum x_{\alpha} ; \quad \alpha, \beta \in G
$$

where $w y_{\beta} \in D(L)_{\lambda+\beta}, x_{\alpha} \in D(L)_{\alpha}$. From this and the fact that the sum of the $D(L)_{\sigma}$ is direct, it follows that for any $\beta \in G w y_{\beta}=x_{\alpha}$ for a suitable $\alpha \in G$. Now choose $\beta \in G$ such that $y_{\beta} \neq 0$. Then $\lambda+\beta=\alpha$ and thus $\lambda=\alpha-\beta \in G$.

We are now in a position to completely describe the structure of $S z(D(L))$.

THEOREM 1.4. Let $\lambda_{1}, \ldots, \lambda_{r}$ be a $\mathrm{Z}$-basis for $\Lambda_{D}(L)$. Choose nonzero semiinvariants $w_{i} \in D(L)_{\lambda_{i}}$. Then the following hold

1. (a) For any $\lambda \in \Lambda_{D}(L)$ we have $D(L)_{\lambda}=Z(D(L)) w_{1}^{m_{1}} \cdots w_{r}^{m_{r}}$, where the integers $m_{i}$ are uniquely determined by $\lambda=m_{1} \lambda_{1}+\cdots+m_{r} \lambda_{r}$.

(b) $w_{1}, \ldots, w_{r}$ are algebraically independent over $Z(D(L))$.

2. $S z(D(L))$ is isomorphic to the group algebra $Z(D(L))\left[\Lambda_{D}(L)\right]$.

3. $S z(D(L))=Z(D(L))\left[w_{1}, \ldots, w_{r}, w_{1}^{-1}, \ldots w_{r}^{-1}\right]$.

In particular, $S z(D(L))$ is Noetherian and its quotient field is $Z(D(L))\left(w_{1}, \ldots, w_{r}\right)$, a pure transcendental extension of $Z(D(L))$.

PROOF. For each $m=\left(m_{1}, \ldots, m_{r}\right) \in \mathbf{Z}^{r}$ we put $w^{m}=w_{1}^{m_{1}} \cdots w_{r}^{m_{r}}$. 
1. (a) If $\lambda=m_{1} \lambda_{1}+\cdots+m_{r} \lambda_{r}$ then $w^{m} \in D(L)_{\lambda}$ and so $\left.Z(D L)\right) w^{m} \subset D(L)_{\lambda}$. On the other hand, let $v \in D(L)_{\lambda}$. Then $v\left(w^{m}\right)^{-1} \in D(L)_{0}=Z(D(L))$ and thus $v \in Z(D(L)) w^{m}$.

(b) (a) and the fact that $S z(D(L))=\bigoplus D(L)_{\lambda}$ show that the $w^{m}, m \in \mathbf{Z}^{r}$, form a basis of $S z(D(L))$ over $Z(D(L))$. In particular they are linearly independent over $Z(D(L))$.

2. Using (1) it is now easy to verify that the map $\phi: Z(D(L))\left[\Lambda_{D}(L)\right] \rightarrow$ $S z(D(L))$ sending $a X_{\lambda}$ into $a w^{m}$, where $a \in Z(D(L))$ and $\lambda=m_{1} \lambda_{1}+\cdots+m_{r} \lambda_{r}$, is an isomorphism of $Z(D(L))$-algebras.

3 . Is a direct consequence of (1).

REMARK 1.5. (a) Let $L^{\infty}$ be the intersection of the ideals $L^{n}$, the lower central series of $L$. We know that $\left[L, L^{\infty}\right]=L^{\infty}$ and that $L^{\infty}$ is the smallest ideal $M$ of $L$ such that $L / M$ is nilpotent. In the next section it is shown that the $w_{i}$ may always be chosen in $Z\left(D\left(L^{\infty}\right)\right) \cap D(L)_{\lambda}$.

(b) (3) of the previous theorem offers a new proof for the factoriality of $S z(D(L))$ since it is a localization of $Z(D(L))\left[w_{1}, \ldots, w_{r}\right]$, which is factorial.

We thank Paul Wauters for allowing us to include the following result.

THEOREM 1.6 (P. WAUTERS). If the semicenter $S z(U(L))$ is Noetherian, then $\Lambda(L)$ is finitely generated.

ProOF. For the sake of notation, we put $R=S z(U(L))$ and if $A$ is an ideal of the semigroup $\Lambda(L)$ then we write $R_{A}=\oplus U(L)_{\lambda}, \lambda \in A$ (see e.g. [9] for generalities on semigroups). Clearly, $R_{A}$ is an ideal of $R$. Moreover, if $A_{1} \subset \cdots \subset A_{n} \subset \cdots$ is a strictly ascending chain of ideals of $\Lambda(L)$, then $R_{A_{1}} \subset \cdots \subset R_{A_{n}} \subset \cdots$ is a strictly ascending chain of ideals of $R$. Since $R$ is Noetherian, $\Lambda(L)$ satisfies ACC on ideals. Put $T=k[\Lambda(L)]$, the monoid ring of $\Lambda(L)$ over $k$, which we view as a $\Lambda_{D}(L)$-graded ring. Clearly, every graded ideal of $T$ is of the form $k[A]$ where $A$ is an ideal of $\Lambda(L)$. Hence $T$ satisfies ACC on graded ideals. Since $\Lambda_{D}(L)$ is a finitely generated abelian group, it follows that $T$ is a Noetherian ring $[\mathbf{1 7}$, p. 612]. Therefore $\Lambda(L)$ is finitely generated [9, Theorem 20.7].

COROLlaRY 1.7. $S z(U(L))$ and $Z(U(L))$ need not be Noetherian.

PROOF. By [8, pp. 320-321] there exists a complex solvable algebraic Lie algebra b of dimension 47 for which $\Lambda(\mathbf{b})$ is not finitely generated. Hence, $S z(U(\mathbf{b}))$ is not Noetherian. Moreover, let $\mathbf{h}$ be the 45 dimensional nilpotent ideal of $\mathbf{b}$ as defined in $\left[8\right.$, p. 320]. Then it is easy to check that $\mathbf{h}$ coincides with $\mathbf{b}_{\Lambda}$, the intersection of all ker $\lambda, \lambda \in \Lambda(\mathbf{b})$. By [6, Theorem 1.19] it follows that $Z(U(\mathbf{h}))=Z\left(U\left(\mathbf{b}_{\Lambda}\right)\right)=$ $S z(U(\mathbf{b}))$, which is not Noetherian. Since $\Lambda(\mathbf{h})=0$, this example also demonstrates that the converse of Theorem 1.6 does not hold.

\section{Properties of the localization $U(L)_{e}$.}

LEMMA 2.1. Let $u$ be a nonzero semi-invariant of $U(L)$ with weight $\lambda$. Then $Z\left(U\left(L^{\infty}\right)\right)$ contains a nonzero semi-invariant $u^{\prime}$ of $U(L)$ with the same weight.

ProOF. We can find ideals $H_{i}$ of $L$ with $\operatorname{dim} H_{i}=i,\left[L, H_{i}\right] \subset H_{i-1}$ and such that $L^{\infty}=H_{d} \subset \cdots \subset H_{i-1} \subset H_{i} \subset \cdots \subset H_{n}=L$. We now distinguish two cases.

(1) $u \in U\left(L^{\infty}\right)$. Clearly $\lambda$ vanishes on $\left[L, L^{\infty}\right]=L^{\infty}$. Hence $[y, u]=0$ for all $y \in L^{\infty}$, which implies that $u \in Z\left(U\left(L^{\infty}\right)\right)$. 
(2) $u \in U\left(H_{i}\right) \backslash U\left(H_{i-1}\right)$ for some $i>d$. Choose $x \in H_{i} \backslash H_{i-1}$. Then $U\left(H_{i}\right)=$ $U\left(H_{i-1}\right)[x]$ and each element of $U\left(H_{i}\right)$ can be written uniquely as a polynomial in $x$ with coefficients in $U\left(H_{i-1}\right)$ (say on the left) [7, 4.4.4]. In particular $u=$ $a_{m} x^{m}+\cdots+a_{1} x+a_{0}$ for some $a_{j} \in U\left(H_{i-1}\right), a_{m} \neq 0$. Take any $y \in L$. Then ad $y(x) \in H_{i-1}$ and $\lambda(y) u=\operatorname{ad} y(u)=\left(\operatorname{ad} y\left(a_{m}\right)\right) x^{m}+$ terms of lower degree in $x$.

It follows that ad $y\left(a_{m}\right)=\lambda(y) a_{m}$ for all $y \in L$, i.e. $a_{m} \in U(L)_{\lambda} \cap U\left(H_{i-1}\right)$. Finally, after repeating the same reasoning a number of times, we find a nonzero semi-invariant $u^{\prime}$ of $U(L)$ with weight $\lambda$, contained in $U\left(L^{\infty}\right)$. Then $u^{\prime} \in Z\left(U\left(L^{\infty}\right)\right)$ as in Case 1.

Because of Theorem 1.3, we are able to use an approach similar to that of [2, 6.7]. Let $\lambda_{1}, \ldots, \lambda_{r}$ be a Z-basis of the group $\Lambda_{D}(L)$. Since $\Lambda_{D}(L)$ is generated by $\Lambda(L)$, there are distinct weights $\mu_{1}, \ldots, \mu_{t} \in \Lambda(L)$ such that

$$
\lambda_{i}=m_{i 1} \mu_{1}+\cdots+m_{i t} \mu_{t}
$$

for some integers $m_{i j}$. By the foregoing lemma, there exist nonzero semi-invariants $e_{i}$ of $U(L)$ with weight $\mu_{i}$, contained in $Z\left(U\left(L^{\infty}\right)\right)$. [By replacing the $\mu_{i}$, we can take the $e_{i}$ to be irreducible as well.] In particular, $e=e_{1} \cdots e_{t} \in Z\left(U\left(L^{\infty}\right)\right)$ is a nonzero semi-invariant of $U(L)$ with weight $\mu=\mu_{1}+\cdots+\mu_{t}$. We denote by $U(L)_{e}$ the localization (which exists) of $U(L)$ with respect to $\left\{1, e, e^{2}, \ldots\right\}$. Next we put

$$
w_{i}=e_{1}^{m_{i 1}} \cdots e_{t}^{m_{i t}}
$$

Clearly, $w_{i} \in Z\left(D\left(L^{\infty}\right)\right) \cap D(L)_{\lambda_{i}}$. Also, $w_{i} e^{p} \in U(L)$ for some nonnegative integer $p$, i.e. $w_{i} \in U(L)_{e}$. Similarly, $w_{i}^{-1} \in U(L)_{e}$.

Theorem 2.2. Put $A=U(L)_{e}, A_{\lambda}=A \cap D(L)_{\lambda}$ and $S z(A)=\bigoplus A_{\lambda}, \lambda \in L^{*}$. Then the following hold:

1. For any $\lambda \in \Lambda_{D}(L)$ we have $A_{\lambda}=Z(A) w_{1}^{m_{1}} \cdots w_{r}^{m_{r}}$, where the integers $m_{i}$ are uniquely determined by $\lambda=m_{1} \lambda_{1}+\cdots+m_{r} \lambda_{r}$.

2. The semicenter $S z(A)$ is isomorphic to the group ring $Z(A)\left[\Lambda_{D}(L)\right]$. Furthermore, $S z(A)=Z(A)\left[w_{1}, \ldots, w_{r}, w_{1}^{-1}, \ldots, w_{r}^{-1}\right]$.

3. $S z(A)=S z(U(L))_{e}$.

4. Both $S z(A)$ and $Z(A)$ are factorial.

5. $Z(A)=\left\{u e^{-m} \mid u \in U(L)_{m \mu}, m \in \mathbf{N}\right\}$.

PROOF. (1) and (2) are proved in the same fashion as in Theorem 1.4.

3. Any $v \in A_{\lambda}$ is of the form $v=u e^{-m}$ where $u \in U(L)$ and $m$ is a nonnegative integer. Clearly, $u=v e^{m} \in U(L) \cap D(L)_{\lambda+m \mu}=U(L)_{\lambda+m \mu}$ and thus $v \in(S z(U(L)))_{e}$. This implies that $S z(A) \subset(S z(U(L)))_{e}$. On the other hand let $x \in(S z(U(L)))_{e}$. Then $x=\left(\sum_{i} x_{i}\right) e^{-p}$ where $x_{i}$ is a semi-invariant of $U(L)$. Hence $x=\sum_{i}\left(x_{i} e^{-p}\right) \in S z(A)$.

4. $S z(A)$ is factorial, being a localization of the factorial ring $S z(U(L))$. Using (2), the group ring $Z(A)\left[\Lambda_{D}(L)\right]$ is factorial and hence so is $Z(A)$ by a result of Gilmer and Parker [1, Theorem 2.6].

5. From the proof of (3) we deduce that

$$
A_{\lambda}=\left\{u e^{-m} \mid u \in U(L)_{\lambda+m \mu}, m \in \mathbf{N}\right\} .
$$

On the other hand, $Z(A)=A_{0}$. This proves (5). We note that $Z(U(L)) \subset Z(A) \subset$ $Z(D(L))$ and $S z(U(L)) \subset S z(A) \subset S z(D(L))$. Next let $E$ be the multiplicative set 
of nonzero semi-invariants of $U(L)$. An important result by Moeglin asserts that each nonzero ideal of $U(L)$ contains an element of $E[\mathbf{1 1}, \mathbf{1 4}]$, i.e. the localization $U(L)_{E}$ is simple. Also, $U(L)_{E}$ and $D(L)$ have the same center and semicenter, since any nonzero semi-invariant of $D(L)$ is a quotient of two elements of $E$.

Corollary 2.3. 1. Put $S=Z(A) \backslash\{0\}$. The localization $A_{S}$ coincides with $U(L)_{E}$.

2. Any nonzero ideal $I$ of $A$ contains an element of $S$.

3. $Z(D(L))$ is the quotient field of $Z(A)$.

ProOF. 1. Take $x \in U(L)_{E}$. Then $x=u v^{-1}$ with $u \in U(L), v \in U(L)_{\lambda}$, $v \neq 0$. By (1) of the previous theorem, $v=c w$ for some nonzero $c \in Z(A)$ and $w \in A$ such that $w^{-1} \in A$. Clearly, $x=u v^{-1}=\left(u w^{-1}\right) c^{-1} \in A_{S}$. This shows that $U(L)_{E} \subset A_{S}$. On the other hand, any $y \in A_{S}$ is of the form $y=a s^{-1}$ where $a \in A \subset U(L)_{E}$ and $s \in S$.

By (5) of the previous theorem, $s=v e^{-m}$ where $v \in E$ and $m$ is a nonnegative integer. Therefore, $s^{-1}=e^{m} v^{-1} \in U(L)_{E}$ and thus $y=a s^{-1} \in U(L)_{E}$.

2. Let $I$ be a nonzero ideal of $A$. Then $I_{S}=\left\{u s^{-1} \mid u \in I, s \in S\right\}$ is a nonzero ideal of the simple algebra $A_{S}$. Hence, $1 \in I_{S}$, i.e. $1=u s^{-1}$ for some $u \in I, s \in S$. So, $s=u \in I$.

3. Clearly, the quotient field of $Z(A)$, i.e. $Z(A)_{S}$, coincides with the center of $A_{S}=U(L)_{E}$, which is $Z(D(L))$.

3. $k$-linear independence of weights. First we recall the following wellknown result [3, §8, Proposition 1].

LEMMA 3.1. Let $V$ be a vector space over $k ; F$ a subfield of $k$ and $W$ a subspace of $V$ over $F$. If $W$ has a basis over $F$ which is also a basis of $V$ over $k$, then $V$ is generated by $W$ over $k$ and every subset of $W$ free over $F$ is also free over $k$.

We thank Theo Moons for pointing out the following

PROPOSITION 3.2. Suppose $L$ has a decomposition of the form $L=S \oplus N \oplus A$, where $S$ is a semisimple Lie subalgebra, $N$ the nil radical of $L$ and $A$ an Abelian Lie subalgebra such that $[A, S]=0$ and with respect to some basis $x_{1}, \ldots, x_{q}$ of $N$ each $\operatorname{ad}_{N} a, a \in A$, has a diagonal matrix, say $\operatorname{diag}\left(\phi_{1}(a), \ldots, \phi_{q}(a)\right), \phi_{i} \in A^{*}$. (Such a decomposition exists for instance if $L$ is almost algebraic and $k$ is algebraically closed [6, proof of Theorem 1.19.3]). Now, let $\lambda \in \Lambda(L)$ be a weight. Then $\lambda$ vanishes on $S \oplus N$ and its restriction $\left.\lambda\right|_{A}=\sum n_{i} \phi_{i}$ for some nonnegative integers $n_{i}$.

ProOF. The first part is obvious since $S=[S, S]$ and each ad $x, x \in N$, acts locally nilpotently on $U(L)$. Next, put $B=S \oplus A$, which is a Lie subalgebra of $L$. Choose a nonzero semi-invariant $u \in U(L)$ with weight $\lambda$. By the Poincaré-BirkhoffWitt theorem, $u$ has a unique expression: $u=\sum u_{m} x_{1}^{m_{1}} \cdots x_{q}^{m_{q}}, u_{m} \in U(B)$, $m=\left(m_{1}, \ldots, m_{q}\right)$.

Take any $a \in A$ and $n=\left(n_{1}, \ldots, n_{q}\right)$ such that $u_{n} \neq 0$. From ad $a(u)=\lambda(a) u$ and the fact that ad $a\left(u_{m}\right)=0$ we deduce $\lambda(a)=\sum n_{i} \phi_{i}(a)$.

We call $L$ algebraic if $L$ is isomorphic to an algebraic Lie algebra of linear transformations. See e.g. $[4, \S 14]$. 
THEOREM 3.3. Let $L$ be algebraic. If $\lambda_{1}, \ldots, \lambda_{r} \in \Lambda_{D}(L)$ are linearly independent over $\mathbf{Z}$, then they are also linearly independent over $k$.

PROOF. Case $1 . k$ is algebraically closed.

Since $L$ is algebraic it is a fortiori almost algebraic $[\mathbf{2 0}, \S 1.3]$ and hence allows a decomposition $L=S \oplus N \oplus A$ as in the previous proposition. Note that $R=N \oplus A$ is the radical of $L$ and $N$ is the nil radical of $R$. Let $W$ denote the $\mathbf{Q}$-subspace of $A^{*}$ generated by the weights $\phi_{1}, \ldots, \phi_{q}$ of ad $A$ on $N$. Since $L$ is algebraic, so is its radical $R[\mathbf{4}$, p. 309]. Moreover, $R$ is completely solvable, as $k$ is algebraically closed. By [12, Theorem 3.3 and Corollary 3.4] there is a basis $a_{1}, \ldots, a_{t}$ for $A$ such that $\phi_{i}\left(a_{j}\right) \in \mathbf{Q}$ for all $i, j$ and $\operatorname{dim}_{\mathbf{Q}} W=\operatorname{dim}_{k} A^{*}=t$. We may assume that $\phi_{1}, \ldots, \phi_{t}, t \leq q$, form a basis for $W$ over $\mathbf{Q}$. Then the rows of the $t \times t$ matrix $P=\left(\phi_{i}\left(a_{j}\right)\right)$ with rational entries are linearly independent over $\mathbf{Q}$ and so $P$ is nonsingular. This implies that the rows of $P$ are also linearly independent over $k$. Therefore $\phi_{1}, \ldots, \phi_{t}$ are linearly independent over $k$ and thus form a basis for $A^{*}$ over $k$. By Lemma 3.1, every subset of $W$ free over $\mathbf{Q}$ is also free over $k$. On the other hand, Proposition 3.2 asserts that

$$
\left\{\left.\lambda\right|_{A} \mid \lambda \in \Lambda(L)\right\} \subset\left\{\sum_{i=1}^{q} n_{i} \phi_{i} \mid n_{i} \in \mathbf{N}\right\}
$$

which implies that $\left\{\left.\lambda\right|_{A} \mid \lambda \in \Lambda_{D}(L)\right\} \subset W$. Since $\lambda_{1}, \ldots, \lambda_{r} \in \Lambda_{D}(L)$ vanish on $N \oplus S$ and are linearly independent over $\mathbf{Z}$ (and hence over $\mathbf{Q}$ ) the same is true of $\left.\lambda_{1}\right|_{A}, \ldots,\left.\lambda_{r}\right|_{A} \in W$. By the preceding observation $\left.\lambda_{1}\right|_{A}, \ldots,\left.\lambda_{r}\right|_{A}$ are linearly independent over $k$ and the same a fortiori holds for $\lambda_{1}, \ldots, \lambda_{r}$.

Case 2 . Let $k$ be arbitrary and denote by $k^{\prime}$ its algebraic closure. Then $\lambda_{1}, \ldots, \lambda_{r}$ can be extended to weights $\lambda_{1}^{\prime}, \ldots, \lambda_{r}^{\prime}$ of $L^{\prime}=L \otimes k^{\prime}$, which is an algebraic Lie algebra over $k^{\prime}$.

$\lambda_{1}^{\prime}, \ldots, \lambda_{r}^{\prime}$ are linearly independent over $\mathbf{Z}$, hence also over $k^{\prime}$ (Case 1). Consequently, $\lambda_{1}, \ldots, \lambda_{r}$ are linearly independent over $k$.

REMARK 3.4. The following example demonstrates that the previous theorem no longer holds in the almost algebraic case. Let $L$ be the Lie algebra over $\mathbf{C}$ with basis $x, u_{1}, u_{2}$ and nonvanishing brackets: $\left[x, u_{1}\right]=(1+i) u_{1},\left[x, u_{2}\right]=(1-i) u_{2} . L$ is a solvable, almost algebraic Lie algebra, $u_{1}, u_{2}$ are semi-invariance with weights $\lambda_{1}, \lambda_{2}$. Clearly $\lambda_{1}, \lambda_{2}$ are linearly independent over $\mathbf{Z}$, but not over $\mathbf{C}$.

\section{REFERENCES}

1. D. F. Anderson, Graded Krull domains, Comm. Algebra 7 (1979), 79-106.

2. W. Borho, P. Gabriel and R. Rentschler, Primideale in Einhüllenden auflösbarer Lie-Algebren, Lecture Notes in Math., vol. 357, Springer-Verlag, Berlin and New York, 1973.

3. N. Bourbaki, Algebra I, Chapter II, Hermann, Paris, 1974.

4. C. Chevalley, Théorie des groupes de Lie, vol. III, Hermann, Paris, 1968.

5. P. M. Cohn, Algebra, vol. 2, Wiley, New York, 1977.

6. L. Delvaux, E. Nauwelaerts and A. I. Ooms, On the semicenter of a universal enveloping algebra, J. Algebra 94 (1985), 324-346.

7. J. Dixmier, Enveloping algebras, North-Holland Mathematical Library, vol. 14, North-Holland, Amsterdam, 1977.

8. J. Dixmier, M. Duflo and M. Vergne, Sur la représentation coadjointe d'un algèbre de Lie, Compositio Math. 29 (1974), 309-323.

9. R. Gilmer, Commutative semigroup rings, Chicago Univ. Press, Chicago, Ill., 1984. 
10. L. Le Bruyn and A. I. Ooms, The semicenter of an enveloping algebra is factorial, Proc. Amer. Math. Soc. 93 (1985), 397-400.

11. M. P. Malliavin, Ultra produit d'algèbres de Lie, Lecture Notes in Math., vol. 924, SpringerVerlag, Berlin and New York, 1982, pp. 157-166.

12. J. C. McConnell, Representations of solvable Lie algebras and the Gelfand-Kirillov conjecture, Proc. London Math. Soc. 29 (1974), 453-484.

13. C. Moeglin, Factorialité dans les algèbres enveloppantes, C. R. Acad. Sci. Paris Ser. A 282 (1976), 1269-1272.

14. __ Idéaux bilatères des algèbres enveloppantes, Bull. Soc. Math. France 108 (1980), 143-186.

15. T. Moons, E. Nauwelaerts and A. I. Ooms, On the weight spaces of Lie algebra modules and their Jordan kernel, J. Algebra 107 (1987), 28-42.

16. T. Moons and A. I. Ooms, On the Jordan kernel of a universal enveloping algebra, J. Algebra (to appear).

17. C. Nðstðsescu and F. Van Oystaeyen, Graded rings with finiteness conditions. II, Comm. Algebra 13 (1985), 605-618.

18. R. Rentschler and M. Vergne, Sur le semi-centre du corps enveloppant d'une algèbre de Lie, Ann. Sci. École Norm. Sup. (4) 6 (1973), 389-405.

19. R. Resco, L. W. Small and A. R. Wadsworth, Tensor products of division rings and finite generation of subfields, Proc. Amer. Math. Soc. 77 (1979), 7-10.

20. L. Auslander and J. Brezin, Almost algebraic Lie algebras, J. Algebra 8 (1968), 295-313.

Department of MAthematics, University of Limburg, LuC. 3610 Diepenbeek, BELGIUM 